\title{
Atomic scale modeling to understand how matter organizes during growth of ultrathin materials in close relation with elaboration process parameters: climbing the scales
}

\author{
A. Hémeryck, ${ }^{1}$ M. Guiltat, ${ }^{1}$ N. Salles, ${ }^{1}$ and N. Richard, ${ }^{2}$ Member, IEEE
}

\begin{abstract}
A multi-levels modeling approach focusing on the elaboration of directly integrated materials is described. Atomic scale calculations are conducted to identify local mechanisms occurring during the growth of material and are then used to parameterize a macroscopic code, developed to simulate vapor like deposition process. The objective is to establish the link between the microstructure of materials and the way they are achieved, i.e. process parameters.
\end{abstract}

\section{INTRODUCTION}

An absolute control at the atomic scale of the material growth during the technological processes, to tailor composition of the layers, and especially to master the formation of interfaces is necessary since this is this atomic arrangement at the nanoscale level which will impact decisively and ensure final properties to the achieved materials. Developing the means of predicting and controlling the structures of integrated materials at the nanometre scale will thus help to push forward their elaboration.

In this context, predictive simulation of the deposition process is strategic. We present a multi-levels approach conducted with the objective to provide an original TCAD tool endowed by an atomistic granularity, as a new tool-forthe-engineer dedicated to the design of tailored integrated material. A multi-levels modeling study is detailed where atomic-scale observations are used as input data to parameterize a macroscopic code, based on a kinetic Monte Carlo methodology. This code is able to simulate vapor-like deposition process and to predict interface composition as a function of applied process parameters. The developed code will help to understand and evaluate the influence of experimental conditions on the material nanostructuring, linking design and manufacturing. In this paper, the deposition of $\mathrm{CuO}$ on $\mathrm{Al}(111)$ substrate is described.

\section{Climbing The ScAles: A Multi-Levels Strategy}

\section{A. Atomistic investigations of the growth local} mechanisms using atomic scale calculations

Massive $a b$ initio calculations are performed to identify local mechanisms at the atomic scale as they could occur during the growth.

A. Hémeryck, M. Guiltat and N. Salles are with LAAS-CNRS, Université de Toulouse, CNRS, UPS, Toulouse, France (corresponding author: Anne Hémeryck +33 5613364 94; fax: +33 5613362 08; e-mail: anne.hemeryck@laas.fr, mguiltat@laas.fr,nsalles@laas.fr).

N. Richard is CEA-DAM-DIF; Bruyères-le-Châtel ; F-91297 Arpajon Cedex, France (e-mail: nicolas.richard@cea.fr).
All the atomic diffusions are thus precisely characterized in terms of energetics (activation barriers and energy gains). All the results presented in this work have been conducted using DFT methodology as implemented into VASP 5.3.3 software.

\section{B. Simulation of the deposition process}

Atomic scale data are then implemented into a homemade code, based on a kinetic Monte Carlo methodology (kMC). This code mixes all the atomic scale events. It simulates $\mathrm{CuO}$ deposition on $\mathrm{Al}(111)$ substrate as a function of realistic process parameters taking into account gases and substrate temperatures, deposition duration, gases pressures... This level of modeling reaches size and time scales characteristic of the process scale [1].

At each step of the kMC cycle, each site of the system is scanned in order to identify all the possible events that can occur in the given state. For each possible event, an occurrence probability is associated. Two kinds of events are defined: Atomic diffusion and Arrival. The occurrence probability of an atomic diffusion event is calculated using the Arrhenius Law formula given in (1):

$$
P_{i}=v \cdot e^{\left(\frac{-E_{i}}{k_{B} T}\right)}
$$

where $v=10^{12} \mathrm{~s}^{-1}$ is the attempt frequency, $\mathrm{E}_{\mathrm{i}}$ is the activation barrier of the diffusion $\mathrm{i}, \mathrm{k}_{\mathrm{B}}$ is the Boltzmann constant and $\mathrm{T}$ is the temperature.

The occurrence probability of an Arrival event is calculated using Boltzmann Statistics given in (2):

$$
P_{i}=C \frac{P S}{\sqrt{M T}}
$$

where $\mathrm{T}$ is the chamber temperature, $\mathrm{P}$ the gas pressure, $\mathrm{M}$ the molar mass, $\mathrm{S}$ the elementary surface unit and $\mathrm{C}$ a constant. Using this approach, we introduce a matter supply into the simulation and the process parameters $\mathrm{P}$ and $\mathrm{T}$, as carried out during deposition.

Then the Poisson formula (3) is used to associated an occurrence time to the occurrence probability as:

$$
t_{i}=t_{\text {simulation }}-\frac{\ln (z)}{P_{i}}
$$

with $\mathrm{z}$ is a random number. The formula introduces a time dependency in the simulation. This time can be seen as the time for one event to be realized. 


\section{RESULTS AND DISCUSSION}

\section{A. Complex chemistry during the formation of interfacial layer}

All these atomic scale results highlight the complexity of the interface formation due to the chemistry of materials themselves (Fig. 1). The adsorption of $\mathrm{CuO}$ on $\mathrm{Al}(111)$ is dissociative. $\mathrm{O}$ atoms exhibit a thermally activated surface diffusion behavior whereas $\mathrm{Cu}$ will diffuse back and forth on the surface due to a non-existing diffusion barrier when isolated. Both $\mathrm{Cu}$ and $\mathrm{O}$ atoms tend to form island on the $\mathrm{Al}(111)$ surface. But while $\mathrm{Cu}$ prefers to penetrate deep into the substrate [2], $\mathrm{O}$ will extract Aluminum atoms [3] to form oxide nucleus. Oxidized part on the surface will trap $\mathrm{Cu}$ species, avoiding them to diffuse into the bulk [4].

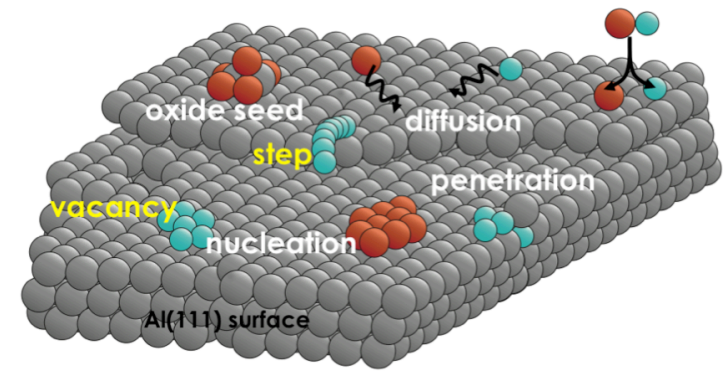

Fig. 1. Schematic of investigated local mechanisms using DFT.

\section{B. Design material elaboration thanks to the control of kinetics at the atomic scale}

We compare simulations of $\mathrm{CuO}$ deposition at low temperature $(120 \mathrm{~K})$ and room temperature $(300 \mathrm{~K})$.

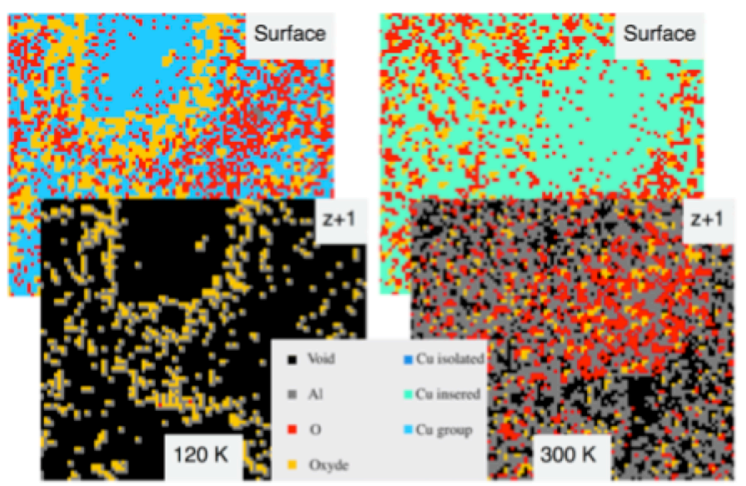

Fig. 2. Simulations of $\mathrm{CuO}$ deposition at $120 \mathrm{~K}$ (left) and $300 \mathrm{~K}$ (right).

At low temperature, $\mathrm{Cu}$ atoms do not succeed to insert into the $\mathrm{Al}(111)$ substrate due to the height of their activation barriers. We can notice on Fig. 2, that a large part of the surface is covered by non-inserted $\mathrm{Cu}$ atoms (in blue) surrounded by oxidized parts (in yellow).

At room temperature, a massive insertion of $\mathrm{Cu}$ atoms is observed into the substrate, leading to rich-copper zone up to 15-20 layers below the surface. Here, oxidized parts are distributed homogeneously on the topmost surface.

We notice here that copper is a key species to control interfacial layer morphology. Notably, using this deposition simulation tool, we can thus evaluate the effect of the temperature on the nanostructuring of the interface. Authorizing copper diffusion thanks to temperature enables to control the thickness and the composition of the interfacial layer.

\section{CONCLUSION}

We propose a computationally-driven approach for the design of material to predict their microstructure and morphology during technological process using a multilevels modeling approach coupling atomic scale understanding of the local mechanisms occurring during the growth and predictive simulations of deposition process. This tool will enable virtual experiments of the deposition to foresee deposition parameters influence on final materials morphology towards a chemically controlled structure of materials and interfaces.

Here, we point out the diversity of the atomic scale diffusions associated to the complex chemistry encountered during the interface formation. We also highlight that applying controlled parameters such as temperature during deposition can lead to tailored interfaces thanks to the atomic scale investigation and understanding, as a promising way to control matter toward dedicated applications.

\section{ACKNOWLEDGMENT}

The computer simulations were performed using HPC resources from CALMIP (Grant 1418).

\section{REFERENCES}

[1] A. Hemeryck, A. Estève, N. Richard, M. Djafari Rouhani, "A kinetic Monte Carlo study of the initial stage of silicon oxidation: basic mechanisms-induced partial ordering of the oxide interfacial layer", Surface Science, vol. 603, pp. 2132-2137, 2009.

[2] L. Marín, C. Nanayakkara, J.F. Veyran, B. Warot-Fonrose, S. Joulie, A. Estève, C. Tenailleau, Y. Chabal and C. Rossi, "Enhancing the reactivity of $\mathrm{Al} / \mathrm{CuO}$ Nanolaminates by $\mathrm{Cu}$ incorporation at the Interfaces", Applied Materials \& Interfaces 7, pp. 11713-11718, 2015.

[3] C. Lanthony, J.M. Ducéré, M. Djafari Rouhani, A. Hémeryck, A Estève and C. Rossi "On the early stage of aluminum oxidation: an extraction mechanism via oxygen cooperation", Journal of Chemical Physics 137, 094707, 2012.

[4] C. Lanthony, M. Guiltat, J.M. Ducéré, A. Verdier, A. Hémeryck, M Djafari Rouhani, C. Rossi, Y. Chabal and A. Estève, "Elementary Surface Chemistry during $\mathrm{CuO} / \mathrm{Al}$ Nanolaminate-thermite Synthesis: Copper and Oxygen Deposition on Aluminum (111) Surfaces", Applied Materials \& Interfaces 6, pp. 15086-15097, 2014. 